\title{
Antifungal Effect of Triglycerol Monolaurate Synthesized by Lipozyme 435-Mediated Esterification
}

\author{
Song Zhang ${ }^{1}$, Jian Xiong ${ }^{1}$, Wenyong Lou' ${ }^{1}$, Zhengxiang Ning ${ }^{1}$, Denghui Zhang ${ }^{2}$, and Jiguo Yang ${ }^{1 *}$ \\ ${ }^{1}$ School of Food Science and Engineering, South China University of Technology, 381 Wushan Road, Guangzhou \\ 510641, P.R. China \\ ${ }^{2}$ Innovation Center of Bioactive Molecule Development and Application, South China Institute of Collaborative \\ Innovation, Xuefu Road, Dongguan 221116, P.R. China
}

This study was designed to synthesize triglycerol monolaurate (TGML) with Lipozyme 435 as the catalyst, and explore its effects on the growth of Aspergillus parasiticus (A. parasiticus) and Aspergillus flavus (A. flavus) and the secretion of aflatoxin b1. The highest content of TGML (49.76\%) was obtained at a molar ratio of triglycerol to lauric acid of 1.08 , a reaction temperature of $84.93^{\circ} \mathrm{C}$, a reaction time of $6 \mathrm{~h}$ and an enzyme dosage of $1.32 \%$. After purification by molecular distillation combined with the washes with ethyl acetate and water, the purity of TGML reached $98.3 \%$. Through characterization by electrospray-ionization mass spectrometry, infrared spectrum and nuclear magnetic resonance, the structure of TGML was identified as a linear triglycerol combined with lauroyl at the end. Finally, the inhibitory effects of TGML on the growths of $A$. parasiticus and $A$. flavus and the secretion of aflatoxin b1 were evaluated by measuring the colony diameter, the inhibition rate of mycelial growth and the content of mycotoxin in the media. The results indicated that TGML had a stronger inhibitory effects on colony growth and mycelial development of both toxic molds compared to sodium benzoate and potassium sorbate, and the secretions of toxins from $A$. parasiticus and $A$. flavus were completely suppressed when adding TGML at 10 and $5 \mathrm{mM}$, respectively. Based on the above results, TGML may be used as a substitute for traditional antifungal agents in the food industry.

Keywords: Triglycerol monolaurate, enzymatic synthesis, purification, antifungal activity, toxin inhibition

Received: October 21, 2019 Accepted: January 16, 2020

First published online: January 23,2020

*Corresponding author Phone: $+86-13560396620$ Fax: +86-0769-38822110 E-mail: yangjg@scut.edu.cn

Supplementary data for this paper are available on-line only at http://jmb.or.kr.

pISSN 1017-7825 elSSN 1738-8872

Copyright(C) 2020 by The Korean Society for Microbiology and Biotechnology

\section{Introduction}

A variety of foods and agricultural products, including oil seeds, grains, tree nuts, and dried fruits, are susceptible to contamination by toxin-producing fungi [1]. Aspergillus, Penicillium and Fusarium are common harmful fungi [2]. They not only cause huge economic losses to the food industry and agriculture [3, 4], but also produce some mycotoxins with hepatotoxicity, nephrotoxicity, immunotoxicity, teratogenicity and carcinogenic capacity $[5,6]$. Among the studied mycotoxins, the most dangerous is aflatoxin b1, which is the main secondary metabolite of Aspergillus parasiticus (A.parasiticus) and Aspergillus flavus (A. flavus) [7]. Due to their heat resistance, aflatoxins on contaminated foods are easily transferred to the diet even if cooked at high temperatures, which poses a great threat to people's health [8]. Therefore, aflatoxin b1 has been classified as a group 1 carcinogen by the International Agency for Research on Cancer [9].

In order to maintain the safety of foods and agricultural products and extend shelf life, medium-chain fatty acids and their monoglycerides have been widely used to control the growth of hazardous fungi and the secretion of mycotoxins [10-12]. For example, Clelia Altieri reported that $20 \mathrm{ppm}$ of lauric acid completely inhibited the growth of Fusarium oxysporum DSMZ 2018 and Fusarium avenaceum DSMZ 62151 on potato dextrose agar within 30 days, which was significantly stronger than palmitic acid, myristic acid and their monoglycerides [12]. However, these fatty acid preservatives are generally water insoluble [13], which severely limits their use in food preservation. Currently, although some researchers have proposed the construction of microemulsions [14], liposomes [15] and nanocapsules [16] to improve their solubility, the usage of these formulations in food is challenged by low stability, high cost and complicated preparation process. Therefore, it is necessary to develop a lipid additive with good water solubility as the substitute for fatty acids and their monoglycerides for food preservation.

Triglycerol mono-fatty acid ester is a kind of polyglycerol ester of fatty acids that has excellent emulsion stability [17] and is recognized by the United States and European Union as a safe substance that can be added to foods [18]. In recent years, the inhibitory activities of polyglycerol monoesters against bacteria and yeast have been reported 
$[19,20]$. We also found that triglycerol monolaurate (TGML) has superior antibacterial activity and inhibitory stability against gram-negative bacteria, gram-positive bacteria and yeasts than that of sodium benzoate (SB) and potassium sorbate (PS) [21]. However, there are few reports on the inhibitory effect of polyglycerol mono-fatty acid esters on toxic molds.

Generally, the syntheses of polyglycerol fatty acid esters mainly involves chemical catalysts [22] or lipases [23]. Specifically, use of the chemical method can easily generate by-products and unpleasant flavors, which leads to difficulties in subsequent separation and purification and makes them unsuitable for use as food additives in the food industry [24]. The enzymatic method is a green preparation process with the advantages of mild reaction conditions and fewer by-products, which has attracted the attention of many researchers. Although some polyglycerol fatty acid esters, such as decaglycerol laurate, oligoglycerol linoleate and polyglycerol polyricinoleate, have been synthesized for use as food emulsifiers using enzyme catalysts recently [23, 25, 26], the preparation of antimicrobial polyglycerol fatty acid esters is different from that of polyglycerol esters used as emulsifiers. According to previous report [27], the antibacterial active ingredient in fatty acid glycerides is mainly monoglyceride, excluding diglyceride and triester. Similarly, it is speculated that polyglycerol monoester is the main antibacterial active ingredient in polyglycerol fatty acid esters based on recent studies [19-21]. However, few studies have reported enzymatic synthesis and yield optimization of polyglycerol monoester.

As we know, the usual purification methods of polyglycerol fatty acid esters involve column chromatography [17] and distillation method [28]. Although column chromatography can provide a high purity product, it usually requires use of a highly toxic reagent such as chloroform, thus the purified product is not suitable as a food additive. Besides, its purification rate is slow and limited to laboratory preparation. As for the distillation method, it is generally difficult to obtain a product of high purity, which severely limits its application in antibacterial experiment. Therefore, it is necessary to develop a new purification method that is low-toxic, fast, and can produce high-purity products on a large scale.

In this study, TGML was synthesized by triglycerol and lauric acid catalyzed by Lipozyme 435 and optimized by response surface test. Then, the crude ester was purified by molecular distillation in combination with ethyl acetate and water washes. The purified TGML was verified by electrospray ionization mass spectrometry (ESIMS), infrared spectrum (IR) and nuclear magnetic resonance (NMR). Finally, the inhibitory effects of TGML on the growth of $A$. parasiticus and A. flavus and the secretion of aflatoxins b1 were explored with comparison to SB and PS.

\section{Materials and Methods \\ Materials}

Glycerol (purity $\geq 99.0 \%$ ), ethanol (purity $\geq 99.7 \%$ ), ethyl acetate (purity $\geq 99.5 \%$ ) and phosphoric acid (purity $\geq 85.0 \%$ ) were purchased from Tianjin Damao Chemical Reagent Co. (China). Lauric acid (purity $\geq$ $98.0 \%$ ), SB (purity $\geq 99.0 \%$ ), PS (purity $\geq 99.0 \%$ ), and sodium hydroxide (purity $\geq 98.0 \%$ ) were obtained from Aladdin Biochemical Technology Company (China). Lipozyme 435 was obtained from Novozymes Co. (Denmark). All water is purified by Mili-Q (Advantage A10, Merck KgaA, Germany).

\section{Strain Cultures}

A. parasiticus ATCC 36537 and A. flavus ATCC 28539 were purchased from Beina Biotechnology Co. (China). After strain activation, two molds were inoculated on potato dextrose agar (PDA). After cultivation at $28^{\circ} \mathrm{C}$ for 5 7 days, the fungal spores were collected in sterile water by scraping the mold colonies with sterile inoculation loop and shaking with several glass drops for $5 \mathrm{~min}$ followed by filtration with double-layer lens paper. Finally, the absorbance of the spore suspension at $600 \mathrm{~nm}$ was adjusted to approximately 0.3 , which corresponded to a spore concentration of $1-5 \times 10^{6} \mathrm{CFU} / \mathrm{ml}$.

\section{Synthesis and Purification of Triglycerol}

The synthesis of triglycerol was carried out according to the method of Bin Peng with some modifications [29]. Glycerol $(500 \mathrm{~g})$ and sodium hydroxide $(10 \mathrm{~g}, 2 \mathrm{wt} \%$ of glycerol) were added into a $2 \mathrm{~L}$ three-necked flask which was heated by a digital magnetic stirring thermostat (KDM, Tianjin Saidlis Experimental Analytical Instrument Manufacturing Co., China). Before heating, nitrogen was passed into the flask to remove air. The reaction is carried out at $260^{\circ} \mathrm{C}$ for $3 \mathrm{~h}$. After the reaction, the temperature dropped to below $100^{\circ} \mathrm{C}$, the reaction product was collected and neutralized with phosphoric acid. Subsequently, 5 volumes of absolute ethanol were added to precipitate the phosphate and then removed by distillation under reduced pressure. Finally, the synthesized triglycerol was purified by two-stage molecular distillation $\left(170^{\circ} \mathrm{C} / 20 \mathrm{~Pa} ; 200^{\circ} \mathrm{C} / 20 \mathrm{~Pa}\right)$ with a scraping speed of $300 \mathrm{rpm}$. The fraction between 170 and $200^{\circ} \mathrm{C}$ was final triglycerol.

The purified triglycerol was analyzed using an HPLC device (Waters e2695, Waters Corp., USA) equipped with a refractive index detector. A Luna $\mathrm{NH}_{2}$ column $(250 \times 4.6 \mathrm{~mm}, 5 \mu \mathrm{m})$ was used with acetonitrile/water $(85 / 15, \mathrm{v} /$ $\mathrm{v})$ as the mobile phase at a flow rate of $1.0 \mathrm{ml} / \mathrm{min}$. The temperatures of column and flow cell were both $30^{\circ} \mathrm{C}$. As shown in Fig. S1 (in supplementary file), the purity of final triglycerol was up to $94.2 \%$. The average degree of polymerization of polyglycerol was detected by the method of REN Chun-fang [30]. After purification by molecular distillation, the average degree of polymerization of the final product was 2.90 , which confirmed that the purified polyglycerol was triglycerol. 
Enzymatic Synthesis of TGML

Triglycerol, lauric acid and Lipozyme 435 were added to a $1 \mathrm{~L}$ three-necked flask, and the reaction was carried out at $300 \mathrm{rpm}$. In the single factor experiment, the percentage of TGML (PT, \%) in crude ester was determined under different molar ratio of triglycerol to lauric acid (2:1-1:2), reaction temperature $\left(60-100^{\circ} \mathrm{C}\right)$, reaction time $(4-8 \mathrm{~h})$ and enzyme dosage $(0.25-2.00 \%)$.

After the reaction, the product was centrifuged at 4,000 $\times \mathrm{g}$ for $10 \mathrm{~min}$ to separate it into three layers. The lower layer contained unreacted triglycerol, which could be used as the raw material for the next synthesis. The intermediate layer contained Lipozyme 435, which could be recovered by filtration. The upper layer was mainly crude triglycerol laurate, and the content of TGML could be determined by an HPLC system equipped with an evaporative light-scattering detector (ELSD). The operating parameters of ELSD were as follows: drift tube temperature $\left(80^{\circ} \mathrm{C}\right)$, nitrogen flow rate $(2.0 \mathrm{~L} / \mathrm{min})$, gain (1) and impactor (on). In addition, a Kinetex C18 column $(250 \times 4.6 \mathrm{~mm}, 5 \mu \mathrm{m})$ was used with acetonitrile/water $(60 / 40, \mathrm{v} / \mathrm{v})$ as the initial mobile phase and gradually changed to $100 \%$ acetonitrile after $5 \mathrm{~min}$. The flow rate was $1.0 \mathrm{ml} / \mathrm{min}$ with a column temperature of $40^{\circ} \mathrm{C}$.

\section{Response Surface Assay}

Based on the results of the single factor experiment, a three-factor, three-level Box-Behnken design was performed to optimize the synthesis of TGML. The factors studied in this assay included the molar ratio of triglycerol to lauric acid, the reaction temperature and the enzyme dosage. The response value was the percentage of TGML in the synthesized triglycerol laurate. The reaction time was set to $6 \mathrm{~h}$ for all assays. Design Expert software (version 11) was used to perform variance and regression analysis of the experimental results.

\section{Purification Method}

The synthesized crude ester was firstly purified by a molecular distillation device (DCH-80, Zhengzhou Ruida Grain and Oil Technology Co., China) with a distillation temperature of $170^{\circ} \mathrm{C}$, a vacuum degree of 20 pa and a scraping speed of $300 \mathrm{rpm}$, and the heavy phase was collected. Then the collected fraction was again treated by molecular distillation at a higher distillation temperature $\left(200^{\circ} \mathrm{C}\right)$ with other parameters unchanged. The light phase was harvested at this stage.

Considering that the parameters of molecular distillation of triglycerol and TGML were similar, which implied that the triglycerol monoester purified by molecular distillation may be doped with triglycerol, further purification was required. After the two molecular distillations, the collected light phase was dispersed in 10 volumes of ethyl acetate, and then washed with 5 volumes of pure water. After standing for $1 \mathrm{~h}$, the upper layer liquid was collected before vacuum distillation to remove ethyl acetate. The remaining component is pure TGML.

\section{Characterization of ESI-MS, IR and NMR}

The purified TGML was initially identified by MS equipped with an ESI source. The operating parameters were as follows: the ion-spray voltage was $3,500 \mathrm{~V}$ with a capillary temperature of $180^{\circ} \mathrm{C}$. Mass spectra with a mass range of $50 \sim 3,000 \mathrm{~m} / \mathrm{z}$ were obtained in positive ion mode. Further, the structure of TGML was characterized by IR according to a previous report [17]. The test range was fixed at 187,500 9,250 $\mathrm{px}^{-1}$ with a resolution of $4 \mathrm{px}^{-1}$. The ratio of signal to noise was 55,000:1 (peak-to-peak). In addition, the structure of TGML was further determined by ${ }^{13} \mathrm{C}$ NMR spectrum. The sample was dissolved in deuterated chloroform, and tetramethylsilane was used as the internal standard with a chemical shift value of 0 .

\section{Inhibition of Colony Growth by TGML}

The inhibitory effect of TGML on the colony growth of A. parasiticus and A. flavus on solid media was studied according to the report by Maristela Martins with some modifications [31]. TGML was added into the PDA media at the following concentrations: $1.25,2.5,5$, and $10 \mathrm{mM}$. The negative control contained no antibacterial agent, while the positive controls contained added SB and PS at concentrations of $1.25,2.5,5$, and $10 \mathrm{mM}$, respectively. Subsequently, 3 pieces of sterile filter paper $(6 \mathrm{~mm}$ in diameter) were evenly placed on each solid PDA plate and immediately inoculated with $2 \mu \mathrm{l}$ of spore suspension $\left(10^{6} \mathrm{CFU} / \mathrm{ml}\right)$. After cultivation at $28^{\circ} \mathrm{C}$ for 2 days, the diameters of the mold colonies on PDA plates were determined in millimeters.

\section{Effect of TGML on Mycelial Growth}

The inhibitory activity of TGML on the mycelial growth of A. parasiticus and A. flavus in liquid media was evaluated according to a previous report with slight modifications [10]. TGML was added to potato dextrose broth (PDB) media to achieve concentrations of $1.25,2.5,5$, and $10 \mathrm{mM}$, respectively. The media contained SB and PS at $1.25,2.5,5$, and $10 \mathrm{mM}$ were used as the positive controls, and the medium without an antibacterial substance was regarded as the negative control. Then, $100 \mu \mathrm{l}$ of the spore suspension $\left(10^{6} \mathrm{CFU} / \mathrm{ml}\right)$ was added to $9.9 \mathrm{ml}$ of the $\mathrm{PDB}$ media, and cultured at $28^{\circ} \mathrm{C}$ for 2 days with $120 \mathrm{rpm}$ shaking. Finally, the mycelia in PDB was filtered and dried to constant weight. The inhibition rate $\left(\mathrm{I}_{\mathrm{R}}, \%\right)$ of TGML on mycelial growth was calculated as follows:

$\mathrm{I}_{\mathrm{R}}(\%)=(W c-W s) / W c \times 100 \%$

where $W s$ represented the dry weight of mycelia from the samples and positive controls, and $W c$ represented the dry weight of mycelia from the negative control. 


\section{Interference of TGML on Mycotoxin Secretion}

The negative controls, positive controls and experimental groups were firstly prepared as described in 2.9. After culturing at $28^{\circ} \mathrm{C}$ for 14 days, the media were filtered and the filtrate was collected. Subsequently, according to the method of Shao, S. [32], the mycotoxin in the filtrate was extracted twice with dichloromethane before the solvent naturally evaporated in a fume hood. The content of aflatoxin b1 was determined by a fluorescence immunoassay analyzer (Guangzhou Yueyang Biotechnology Co., China). The final toxin content was expressed as $\mu \mathrm{g} / \mathrm{ml}$.

\section{Statistical Analysis}

The data were expressed as the averages \pm standard derivation (SD) of three determinations. The statistical comparison was performed by one-way ANOVA followed by Dunnett's multiple comparisons test in GraphPad Prism 6.00. Different letters were used to represent the significant differences in statistics when $p \leq 0.05$.

\section{Results and Discussion}

\section{Effect of Reaction Conditions on the Production of TGML}

The influence of molar ratio of triglycerol to lauric acid, reaction temperature, reaction time and enzyme dosage on the production of TGML was shown in Figure 1. The content of TGML increased obviously with increase of the molar ratio of triglycerol/lauric acid from 1:2 to 1:1, and the highest content of TGML (47.20 $\pm 1.66 \%$ ) occurred at 1:1 (Fig. 1A). When the molar ratio exceeded 1:1, the content of TGML declined rapidly, probably because the remaining triglycerol was not esterified. A similar effect could also be seen in Figure 1B. The content of TGML gradually increased with an increase in reaction temperature from 60 to $90^{\circ} \mathrm{C}$, and the maximum PT $(46.97 \pm 2.56 \%)$ was acquired at the temperature of $90^{\circ} \mathrm{C}$. However, further increasing temperature to $100^{\circ} \mathrm{C}$ would result in a decrease in the yield of target product, which may be related to the loss of lipase activity and the decrease of its affinity for the substrate under high temperature conditions [33].

As shown in Fig. 1C, the content of TGML significantly increased as the reaction time increased from 4 to $6 \mathrm{~h}$, and then reached a stable phase in the range of $6-8 \mathrm{~h}$. PT reached its maximum $(46.83 \pm 2.47 \%)$ at $6 \mathrm{~h}$. As for Fig. 1D, it was observed that PT increased from $17.17 \pm 1.88$ to $47.40 \pm 2.10 \%$ with increase of the enzyme dosage from 0.4 to $1.2 \%$, and then remained unchanged at the dose range of 1.2 to $2.0 \%$. The highest content of TGML $(47.40 \pm 2.10 \%)$ appeared at the lipase dosage of $1.2 \%$.

Similar studies on the effect of reaction conditions on the synthesis of polyglycerol fatty acid esters by single factor experiment have also been reported. For example, Bin Peng studied the esterification synthesis of polyglycerol and rice-bran oil, C. camphora seed oil, or acetic acid catalyzed by Lipozyme 435 , and found that the best esterification efficiency appeared in the substrate molar ratio of 1.5:1 [29]. In addition, in another lipasecatalyzed transesterification of decylglycerol and methyl laurate, the highest conversion of methyl laurate was observed at a substrate molar ratio of 2:1, a reaction temperature of $65^{\circ} \mathrm{C}$, and an enzyme concentration of $7 \%$ [23].
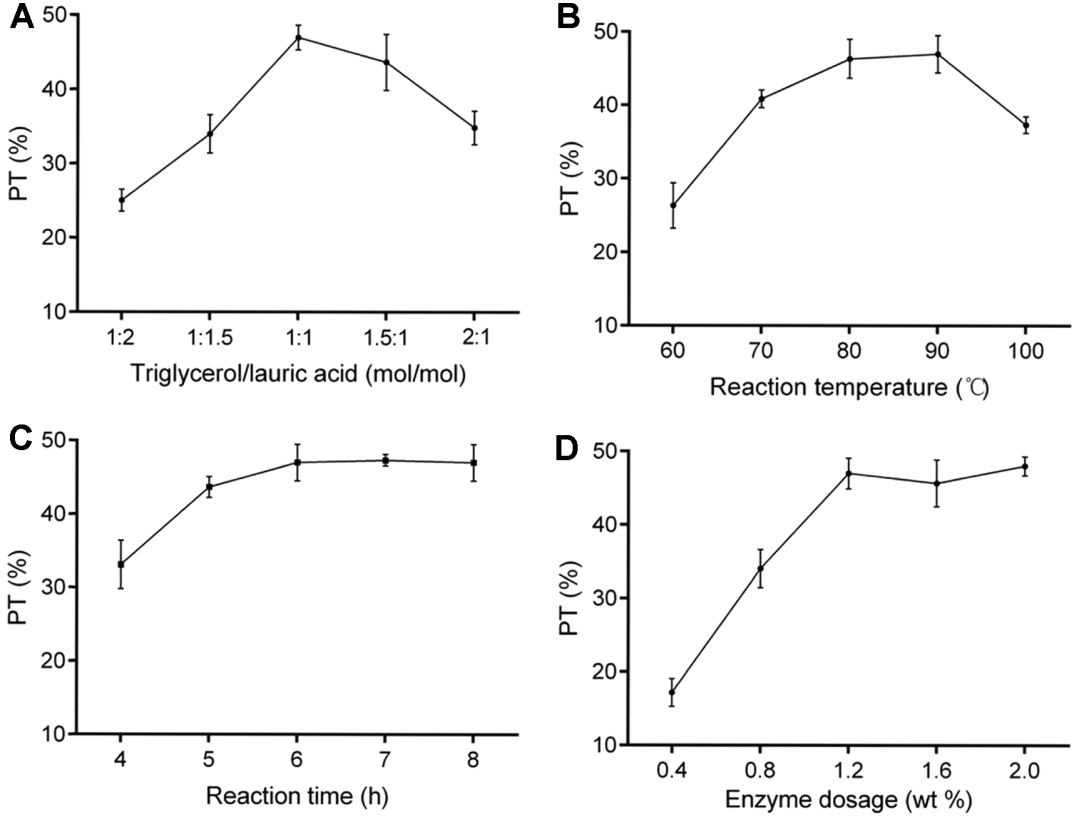

Fig. 1. Single factor tests of enzymatic catalyzed esterification of triglycerol and lauric acid. Percentage of TGML (PT, averages $\pm \mathrm{SD}, n=3$ ) at different $(\mathbf{A})$ molar ratios of triglycerol to lauric acid (reaction temperature, $80^{\circ} \mathrm{C}$; reaction time, $6 \mathrm{~h}$; enzyme dosage, 1.2\%), (B) reaction temperatures (molar ratio of triglycerol to lauric acid, 1:1; reaction time, $6 \mathrm{~h}$; enzyme dosage, $1.2 \%$ ), (C) reaction times (molar ratio of triglycerol to lauric acid, $1: 1$; reaction temperature $80^{\circ} \mathrm{C}$; enzyme dosage, $1.2 \%$ ) and (D) enzyme dosages (molar ratio of triglycerol to lauric acid, $1: 1$; reaction temperature $80^{\circ} \mathrm{C}$; reaction time, $6 \mathrm{~h}$ ). 
Table 1. A three-factor and three-level response surface experiment design (Box-Behnken) of TGML synthesis.

\begin{tabular}{ccccc}
\hline \multirow{2}{*}{ Experiment numbers } & \multicolumn{2}{c}{ Factors } & \\
\cline { 2 - 4 } & Molar ratio $(\mathrm{mol} / \mathrm{mol})$ & Reaction temperature $\left({ }^{\circ} \mathrm{C}\right)$ & Enzyme dosage $(\%)$ & 0.8 \\
2 & $1: 1.5$ & 80 & 1.6 & 30.49 \\
3 & $1.5: 1$ & 80 & 1.6 & 35.63 \\
4 & $1: 1$ & 90 & 0.8 & 47.92 \\
5 & $1.5: 1$ & 80 & 1.2 & 24.52 \\
6 & $1: 1.5$ & 90 & 0.8 & 42.67 \\
7 & $1: 1$ & 90 & 1.2 & 33.16 \\
8 & $1: 1$ & 80 & 0.8 & 46.27 \\
9 & $1: 1$ & 70 & 1.2 & 28.78 \\
10 & $1.5: 1$ & 70 & 1.6 & 29.34 \\
11 & $1: 1$ & 70 & 1.2 & 40.86 \\
12 & $1: 1$ & 80 & 1.2 & 47.13 \\
13 & $1: 1$ & 80 & 1.2 & 48.29 \\
14 & $1: 1$ & 80 & 1.2 & 47.58 \\
16 & $1: 1.5$ & 70 & 1.2 & 37.80 \\
17 & $1.5: 1$ & 90 & 1.6 & 34.69 \\
\end{tabular}

The difference reported by the above literature may be because of the different reaction substrates, esterification methods and detection indicators.

\section{Model Fitting and Response Surface Optimization}

The response surface design (Box-Behnken) of the esterification of TGML was displayed in Table 1. After fitting with the intercept model, the F-value and $\mathrm{R}^{2}$ in ANOVA analysis were 227.81 (at $p<0.0001$ ) and 0.9966 , respectively, indicating that this fitting could correctly reflect the interactions among the three factors (Table 2). The lack of fit test was not significant (F-value of 0.068 at $p>0.05$ ), which further showed that the model fitted the experimental results well. The high fitting coefficient $\left(R^{2}\right.$ of 0.9966 , adjusted $R^{2}$ of 0.9922$)$ suggested that the model could accurately reflect the trend of experimental data. Based on the F value corresponding to each factor, an order of importance of three factors was obtained as follows: enzyme dosage > substrate molar ratio > reaction temperature. The second-order fitting formula for PT calculation was shown as the following:

$\mathrm{PT}(\%)=46.72-3.87 \mathrm{~A}+2.72 \mathrm{~B}+6.44 \mathrm{C}+0.09 \mathrm{AB}-0.20 \mathrm{AC}+0.67 \mathrm{BC}-7.29 \mathrm{~A}^{2}-3.31 \mathrm{~B}^{2}-6.22 \mathrm{C}^{2}$

where $\mathrm{A}$ was the molar ratio of triglycerol to lauric acid, $\mathrm{B}$ was the reaction temperature, and $\mathrm{C}$ was the enzyme dosage.

The influence of the interaction between substrate molar ratio, reaction temperature and enzyme dosage on PT was recorded in Fig. 2. PT was influenced in the following order: $\mathrm{BC}>\mathrm{AC}>\mathrm{AB}$. The optimized conditions for TGML synthesis calculated by Design Expert software were the triglycerol/lauric acid molar ratio of 1.08, the reaction temperature of $84.93^{\circ} \mathrm{C}$, the reaction time of $6 \mathrm{~h}$ and the enzyme dosage of $1.32 \%$. The predicted PT was up to $49.76 \%$. Small differences occurred between the optimized conditions and the actual optimal parameters, which may be derived from the interaction among the three factors. Further, we synthesized TGML again under the optimal conditions, and the PT was $49.34 \%$, which proved the accuracy and reliability of the fitted model.

Table 2. ANOVA analysis for quadratic model.

\begin{tabular}{lcccccc}
\hline \multicolumn{1}{c}{ Source } & Sum of squares & df & Mean square & F-value & $p$-value \\
\hline Model & 1024.88 & 9 & 113.88 & 227.81 & $<0.0001$ & Significant \\
A- Molar ratio of triglycerol to lauric acid & 120.05 & 1 & 120.05 & 240.16 & $<0.0001$ \\
B- Reaction temperature & 57.84 & 1 & 57.84 & 115.72 & $<0.0001$ \\
C- Enzyme dosage & 324.94 & 1 & 324.94 & 650.05 & $<0.0001$ \\
AB & 0.0309 & 1 & 0.0309 & 0.0618 & 0.8109 \\
AC & 0.1630 & 1 & 0.1630 & 0.3260 & 0.5859 \\
BC & 1.80 & 1 & 1.80 & 3.59 & 0.0999 \\
$\mathrm{~A}^{2}$ & 200.70 & 1 & 200.70 & 401.50 & $<0.0001$ \\
$\mathrm{~B}^{2}$ & 46.01 & 1 & 46.01 & 92.05 & $<0.0001$ \\
$\mathrm{C}^{2}$ & 162.81 & 1 & 162.81 & 325.70 & $<0.0001$ \\
Residual & 3.50 & 7 & 0.4999 & & 0.6448 \\
Lack of fit & 1.09 & 3 & 0.3648 & 0.6068 & Not significant \\
Pure error & 2.40 & 4 & 0.6012 & & \\
Cor total & 1028.38 & 16 & & & \\
$\mathrm{R}^{2}$ & 0.9966 & & & &
\end{tabular}



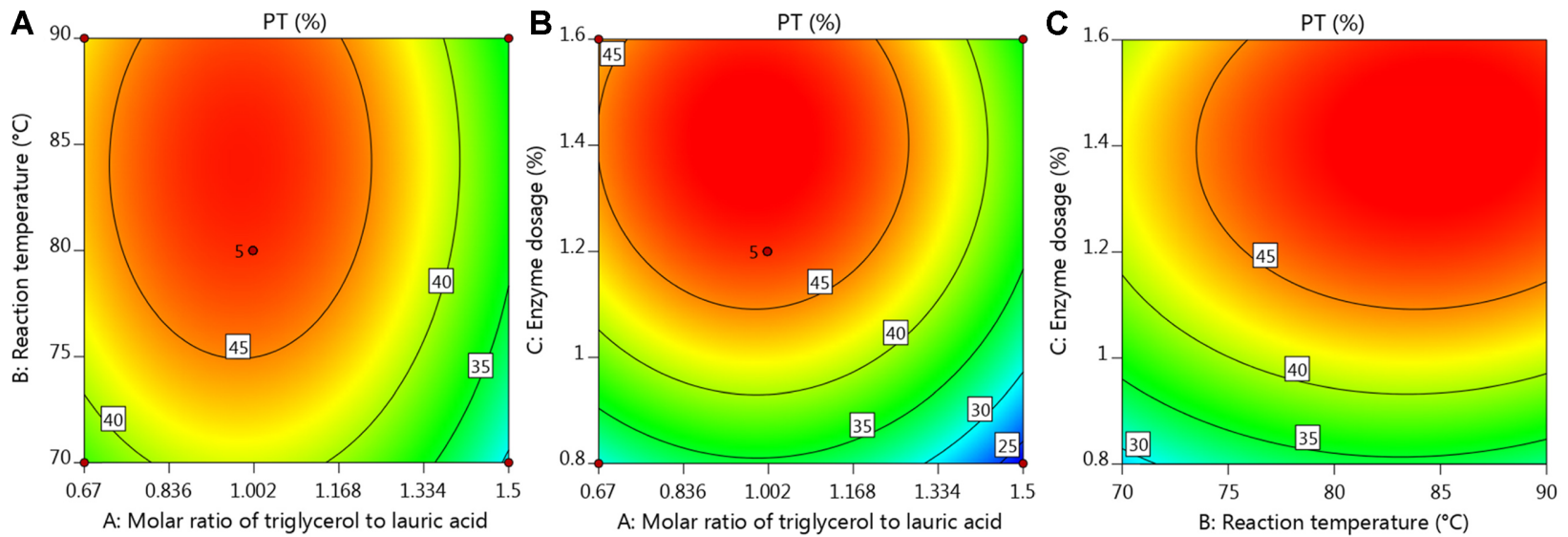

Fig. 2. Contour plots of the interactions among the molar ratio of triglycerol to lauric acid, reaction temperature and enzyme dosage. PT as a function of (A) triglycerol/lauric acid molar ratio and reaction temperature with the enzyme dosage set to 1.2\%, (B) triglycerol/lauric acid molar ratio and enzyme dosage with the reaction temperature set to $80^{\circ} \mathrm{C}$, and $(\mathrm{C})$ reaction temperature and enzyme dosage with the triglycerol/ lauric acid molar ratio set to 1.00

Recently, many studies have also reported the optimization of the synthesis of polyglycerol fatty acid esters. For instance, Bin Peng obtained the best parameters (reaction temperature of $85^{\circ} \mathrm{C}$, reaction time of $6 \mathrm{~h}$, enzyme dosage of $1.4 \%$ and substrate molar ratio of 1.35 ) for esterification of polyglycerol and rice-bran oil through a three-factor, three-level Box-Behnken test, and the predicted esterification efficiency was $69.82 \%$ [29]. Similarly, in another report, the esterification of oligoglycerol with linoleic acid was also optimized by Box-Behnken assay, and the maximum esterification efficiency $(96.15 \%)$ occurred in the reaction time of $4.52 \mathrm{~h}$, the reaction temperature of $90^{\circ} \mathrm{C}$, the enzyme dosage of $2 \%$, the molar ratio of oligoglycerol to linoleic acid of 1.59:1 and no water addition [26]. In addition, an orthogonal test was also used to optimize the synthesis of decaglycerol laurates, and the highest conversion of methyl laurate reached $83.3 \pm 1.5 \%$ under optimal conditions (reaction time $4.5 \mathrm{~h}$, rotating speed $180 \mathrm{rpm}$, enzyme dosage $8 \%$, and water content 5\%) [23]. Compared to the above reports, the response value in this study was lower, probably because the optimization object of this study is a component in the final product.
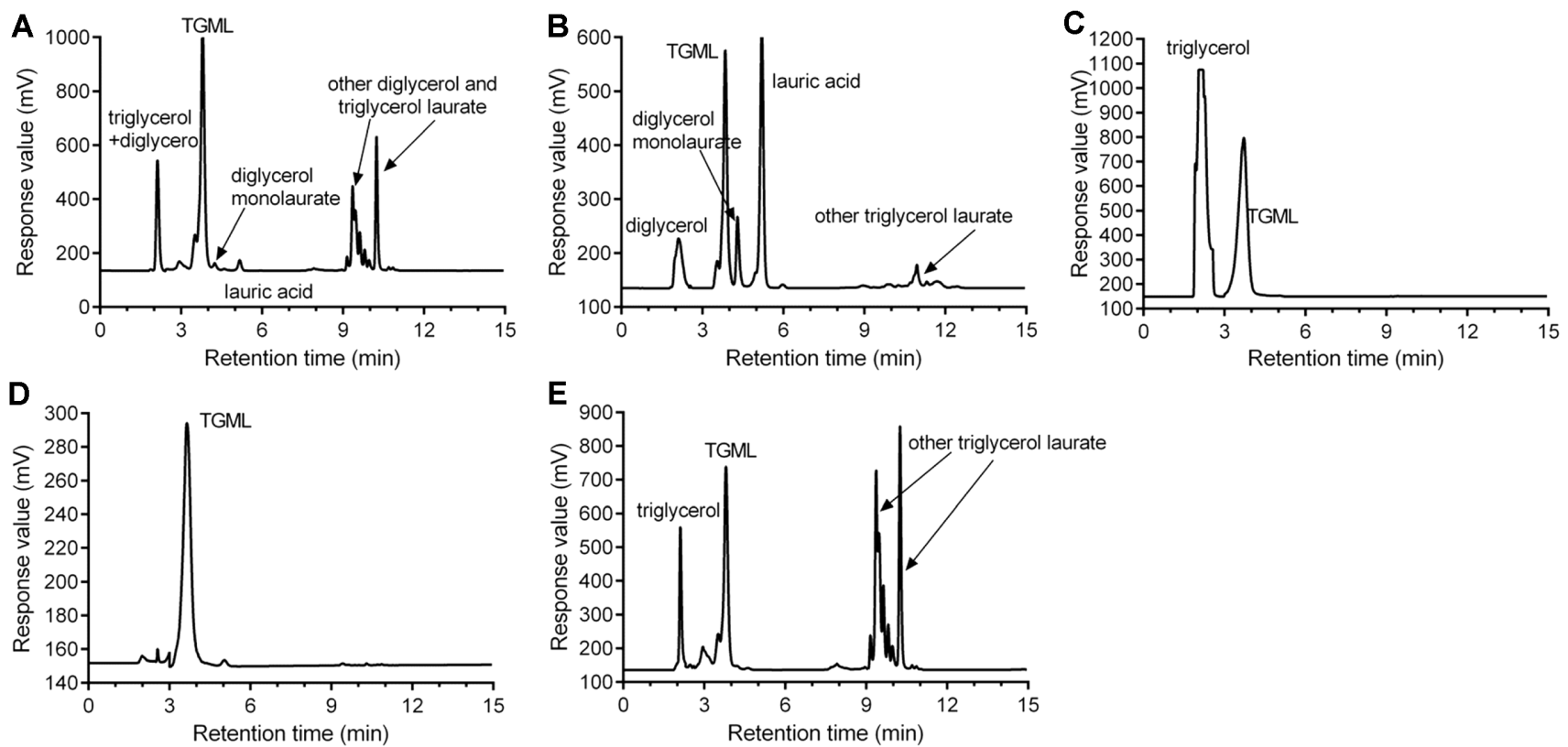

Fig. 3. HPLC chromatograms of synthetic crude ester (A) and the light phase (B), intermediate phase (C), intermediate phase followed by ethyl acetate and water wash $(\mathrm{D})$ and heavy phase $(\mathrm{E})$ separated by molecular distillation at $170-220^{\circ} \mathrm{C}$. 


\section{Purification of TGML}

The synthesized triglycerol laurate was purified by molecular distillation combined with ethyl acetate and water washes (Fig. 3). Based on the determination of HPLC-ELSD, the crude ester contained di- and tri-glycerol (9.58\%), TGML (47.34\%), diglycerol monolaurate (1.31\%), lauric acid $(2.70 \%)$ and other diglycerol and triglycerol laurate (36.22\%) (Fig. $3 \mathrm{~A}$ ). After purification by two-stage molecular distillation $\left(170^{\circ} \mathrm{C} / 20 \mathrm{pa}, 200^{\circ} \mathrm{C} /\right.$ $20 \mathrm{pa}$ ), the middle-phase fraction contained only triglycerol and TGML (Fig. 3C). Subsequently, the triglycerol in the ester was removed by the extraction of TGML by ethyl acetate and the dissolution of triglycerol by water, and the purity of final TGML was up to $98.3 \%$ (Fig. 3D).

Currently, column chromatography and distillation technique are often used in the purification of polyglycerol fatty acid esters. For instance, TGML crude ester was purified to $98.7 \%$ through a silica gel column using chloroform/methanol (90:10) as the eluting solvent [17]. Although the reported purity was very similar to the results in this study, the disadvantages are obvious, such as the use of chloroform (highly toxic) and very slow purification rate. In addition, J. Holstborg reported that diglycerol monoester could be purified to more than $80 \%$ by laboratory distillation process [28]. Obviously, its purity could not meet the requirements of many scientific assays, including antibacterial test.

\section{Characterization of ESI-MS, IR and NMR}

The composition of purified TGML characterized by ESI-MS was shown in Table 3. The purified TGML contained [lauric acid + monoester of triglycerol $+\mathrm{Na}]^{+}(1 \mathrm{H})(100 \%)$, [lauric acid + monoester of triglycerol + $\mathrm{Na}]^{+}(2 \mathrm{H})(19.9 \%)$, [lauric acid + monoester of triglycerol $\left.+\mathrm{Na}\right]^{+}(3 \mathrm{H})(2.9 \%)$, [lauric acid + monoester of tetraglycerol $\left.+\mathrm{Na}-\mathrm{H}_{2} \mathrm{O}\right]^{+}(2.0 \%)$, [lauric acid + diester of triglycerol $\left.+\mathrm{Na}\right]^{+}(0.8 \%)$ and [lauric acid + triester of tetraglycerol $\left.+\mathrm{Na}-\mathrm{H}_{2} \mathrm{O}\right]^{+}(1.1 \%)$. As the dehydration reaction did not occur during the electrospray ionization process [34], it was speculated that the removal of water molecules in the above positive ions might be due to etherification of the adjacent hydroxyl groups in polyglycerol. Based on the ion intensity, the TGML containing three hydrogen isotopes was the most abundant, which was consistent with the result of purified TGML analyzed by HPLC-ELSD.

The functional groups of TGML determined by IR were shown in Fig. S2 (in supplementary file). C=O $\left(1,738 \mathrm{~cm}^{-1}\right)$, C-O-C(symmetrical and asymmetrical stretching vibration at 1,045 and $1,116 \mathrm{~cm}^{-1}$ ) and $\mathrm{OH}$ $\left(3,378 \mathrm{~cm}^{-1}\right)$ illustrated the presence of an ester bond, an ether bond and a hydroxyl group in TGML, respectively. This was in agreement with the results of HPLC-ELSD assay.

In addition, the results of ${ }^{13} \mathrm{C}$ NMR spectra of TGML were shown in Fig. S3 (in supplementary file). It was found that the signals on the triglycerol carbon skeleton were located at 60-72 ppm, and no signal exceeded $80 \mathrm{ppm}$. Therefore, based on the previous reports [34,35], the triglycerol skeleton was judged as linear triglycerol. Besides, referring to the chemical shifts of terminal carbon $(62.92 \mathrm{ppm})$ and intermediate carbon $(70.83 \mathrm{ppm})$ of polyglycerol reported by Fenlong Wan [35], the chemical shifts of terminal and intermediate carbons on the polyglycerol backbone of TGML in this study were $64.00,62.63 \mathrm{ppm}$ and $67.55,68.69,69.90 \mathrm{ppm}$, respectively. The downfield chemical shift showed an occurrence of typical acylation reaction, indicating that lauric acid was mainly bound to the terminal hydroxyl group of triglycerol in enzymatic esterification, which could be explained by the higher reactive activity of primary hydroxyl groups than secondary hydroxyl groups due to the space hindrance effect [36]. Therefore, we concluded that the structure of purified TGML was mainly linear triglycerol combined with lauroyl at the end.

\section{Inhibition of TGML on Colony and Mycelial Growths of Molds}

The inhibition of TGML on the growth of A. parasiticus and A. flavus colonies was clearly seen in Fig. 4. After treatment with TGML at $1.25,2.5$, and $5 \mathrm{mM}$, the colony areas of both molds were significantly reduced, even less than the same concentrations of SB and PS. Further, the antifungal effect of TGML was quantified by measuring the diameters of two Aspergillus colonies. As shown in Table 4, compared to the control $(25.60 \pm 0.60 \mathrm{~mm})$, the diameters of the colonies of $A$. parasiticus dropped significantly to $21.00 \pm 0.20,17.87 \pm 0.61$, and $15.20 \pm$ $0.20 \mathrm{~mm}$ in the treatment of TGML at $1.25,2.5$, and $5 \mathrm{mM}$, respectively, smaller than the colony sizes treated by the same concentrations of SB and PS. As for A. flavus, the inhibitory effect of TGML was more obvious. After adding TGML into the solid media at 1.25, 2.5, and $5 \mathrm{mM}$, the colony diameters of A. flavus would decrease by $8.53,11.20$, and $13.66 \mathrm{~mm}$, respectively, which was also superior to the inhibitory effects of SB and PS.

In addition, the effects of TGML on the $\mathrm{I}_{\mathrm{R}}$ of mycelial growths of two toxic molds in liquid media were shown in Table 5. As the concentration of TGML increased from 1.25 to $10 \mathrm{mM}$, its $I_{R}$ on the mycelial growth of A. parasiticus increased from $12.57 \pm 0.41$ to $91.58 \pm 1.03 \%$. In other words, the mycelial growth of toxic mold was almost totally inhibited at $10 \mathrm{mM}$. However, the maximum $\mathrm{I}_{\mathrm{R}}$ values of $\mathrm{SB}$ and PS on the mycelial growth of this

Table 3. ESI-MS analysis of purified TGML.

\begin{tabular}{cccc}
\hline No. & $\mathrm{m} / \mathrm{z}$ & \multicolumn{1}{c}{ Component } & Intensity $(\%)$ \\
\hline 1 & 445.2782 & [lauric acid+monoester of triglycerol+Na] ${ }^{+}(1 \mathrm{H})$ & 100 \\
2 & 446.2818 & [lauric acid+monoester of triglycerol+Na] ${ }^{+}(2 \mathrm{H})$ & 19.9 \\
3 & 447.2827 & [lauric acid+monoester of triglycerol+Na] ${ }^{+}(3 \mathrm{H})$ & 2.9 \\
4 & 501.3044 & [lauric acid+ monoester of tetraglycerol+Na? $\left.\mathrm{H}_{2} \mathrm{O}\right]^{+}$ & 2.0 \\
5 & 627.4450 & [lauric acid+ diester of triglycerol+Na] & 0.8 \\
6 & 867.5663 & [lauric acid+ triester of tetraglycerol+Na? $\mathrm{H}_{2} \mathrm{O}^{+}$ & 1.1 \\
\hline
\end{tabular}


A

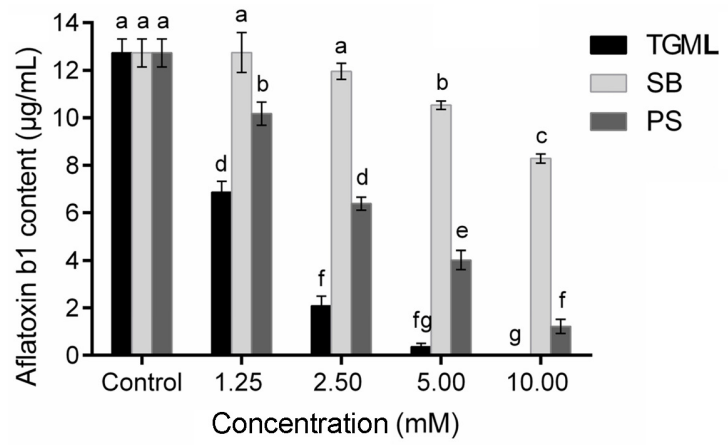

B

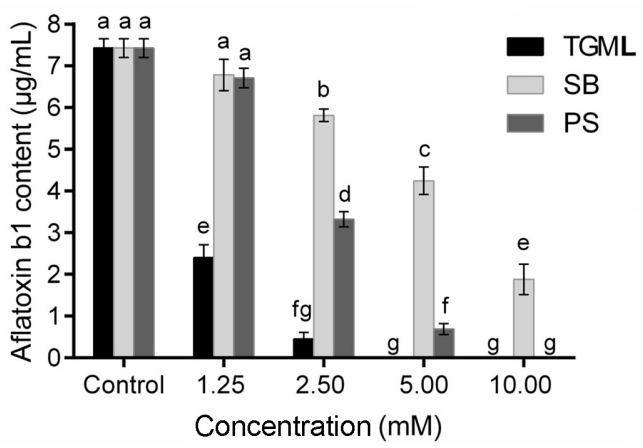

Fig. 4. Content of aflatoxin b1 produced by A.parasiticus (A) and A. flavus (B) treated with TGML, SB and PS at $1.25,2.5,5$ and $10 \mathrm{mM}$. Columns not having the same letters are considered significantly different $(p \leq 0.05)$.

mold were only $56.43 \pm 1.24 \%$ and $62.57 \pm 0.21 \%$ in the concentration range of 1.25 to $10 \mathrm{mM}$. In the test of A. flavus, compared to the $1.25,2.5,5$, and $10 \mathrm{mM}$ of SB and PS, the $\mathrm{I}_{\mathrm{R}}$ of mycelial growth by the same concentrations of TGML increased by $10.37 \%, 18.91 \%, 26.65 \%, 42.81 \%$, and $8.87 \%, 16.88 \%, 21.09 \%, 40.13 \%$, respectively. Besides, it was found that the inhibitory effect of TGML on A. parasiticus in liquid media was close to that of $A$. flavus due to their similar $\mathrm{I}_{\mathrm{R}}$ values.

From the above results, it was concluded that the inhibitory activity of TGML against $A$. parasiticus and A. flavus in the media was obviously stronger than that of SB and PS, which might be related to the effects on the formation of hyphae or pseudohyphae [19]. In addition, its inhibitory effect on toxigenic fungi was concentration dependent, which was similar to the results reported by the recent antifungal studies [10-12, 19,20]. For example, the growths of Saccharomyces cerevisiae, Aspergillus niger and Penicillium glaucum were completely inhibited by monolaurin at $0.32,0.32$, and $0.16 \mathrm{mg} / \mathrm{ml}$, respectively, while the cells or spores of the three fungi were totally removed only when the concentration of monolaurin increased to $1.25,2.5$, and $0.63 \mathrm{mg} / \mathrm{ml}$, respectively [11], indicating that the antifungal effect of monolaurin was directly related to its concentration. Besides, Chikako Ikegawa found that the cell numbers of Saccharomyces cerevisiae decreased by $0,2.2$, and $4.3 \log \mathrm{CFU} / \mathrm{ml}$, respectively, when incubated with diglycerol monolaurate at 31.3,62.5, and $125 \mu \mathrm{g} / \mathrm{ml}$ [20], suggesting that diglycerol monolaurate also exerted a lethal effect against yeast cells by a concentration-dependent manner.

\section{Interference of TGML on Mycotoxin Secretion}

The effect of TGML on the production of aflatoxin b1 produced by two toxic molds was shown in Fig. 4 . In the test of $A$. parasiticus, when cultured with TGML at $1.25,2.5,5$, and $10 \mathrm{mM}$, the amount of aflatoxin b1 secreted by A. parasiticus would decrease from $12.73 \pm 0.59 \mu \mathrm{g} / \mathrm{ml}$ (in the control) to $6.86 \pm 0.46,2.08 \pm 0.41,0.36 \pm 0.15$, and $0.00 \mu \mathrm{g} / \mathrm{ml}$, respectively, lower than the toxin content treated by SB and PS at the same concentrations (Fig. 5A). Similar trends were also observed in the inhibitory assay of A. flavus (Fig. 5B). The content of aflatoxin b1 declined rapidly to 0 as the concentration of TGML increased from 1.25 to $5 \mathrm{mM}$. Compared to SB and PS at the same concentrations, the inhibitory ability of TGML on the secretion of toxin from A. flavus was obviously stronger. Besides, it was found that TGML inhibited the secretion of toxin from A. flavus stronger than that from A. parasiticus, which might be related to the species differences between the two molds.

Recently, many studies on the inhibition of mycotoxin secretion by esters have been reported $[1,6,37,38]$. Tiago M. Nazareth reported that the content of aflatoxin would decrease by $19.9,45.29$, and 100\%, respectively, when

Table 4. Colony diameters of $A$. parasiticus and A. flavus grown on PDA supplemented with TGML, SB, and PS at $1.25,2.5$ and $5 \mathrm{mM}$.

\begin{tabular}{lll}
\hline & & Colony diameter $(\mathrm{mm})$ \\
\cline { 2 - 3 } & A. parasiticus & A. flavus \\
\hline Control & $25.60 \pm 0.60 \mathrm{a}$ & $25.33 \pm 0.31 \mathrm{a}$ \\
TGML $1.25 \mathrm{mM}$ & $21.00 \pm 0.20 \mathrm{~b}$ & $16.80 \pm 0.20 \mathrm{c}$ \\
TGML $2.5 \mathrm{mM}$ & $17.87 \pm 0.61 \mathrm{c}$ & $14.13 \pm 0.42 \mathrm{~d}$ \\
TGML $5 \mathrm{mM}$ & $15.20 \pm 0.20 \mathrm{~d}$ & $11.67 \pm 0.31 \mathrm{e}$ \\
SB $1.25 \mathrm{mM}$ & $25.60 \pm 0.87 \mathrm{a}$ & $25.93 \pm 0.70 \mathrm{a}$ \\
SB $2.5 \mathrm{mM}$ & $24.27 \pm 0.50 \mathrm{a}$ & $24.80 \pm 0.20 \mathrm{a}$ \\
SB $5 \mathrm{mM}$ & $22.53 \pm 0.42 \mathrm{~b}$ & $23.13 \pm 0.31 \mathrm{ab}$ \\
PS $1.25 \mathrm{mM}$ & $25.13 \pm 0.76 \mathrm{a}$ & $22.33 \pm 0.50 \mathrm{~b}$ \\
PS $2.5 \mathrm{mM}$ & $22.60 \pm 0.72 \mathrm{~b}$ & $20.13 \pm 0.42 \mathrm{bc}$ \\
PS $5 \mathrm{mM}$ & $19.33 \pm 0.58 \mathrm{c}$ & $16.27 \pm 0.42 \mathrm{~d}$ \\
\hline
\end{tabular}

The mean values that are not followed by the same letter are significantly different $(p \leq 0.05)$. 
Table 5. Inhibition rates (\%) of mycelial growth of A. parasiticus and A. flavus grown in PDB supplemented with TGML, SB and PS at $1.25,2.5,5$, and $10 \mathrm{mM}$.

\begin{tabular}{lcc}
\hline & \multicolumn{2}{c}{ Inhibition rate of mycelial growth (\%) } \\
\cline { 2 - 3 } & A.parasiticus & A. flavus \\
\hline Control & 0 & 0 \\
TGML $1.25 \mathrm{mM}$ & $12.57 \pm 0.41 \mathrm{a}$ & $33.12 \pm 2.04 \mathrm{ab}$ \\
TGML $2.5 \mathrm{mM}$ & $27.78 \pm 0.41 \mathrm{~b} \mathrm{bc}$ \\
TGML $5 \mathrm{mM}$ & $57.89 \pm 2.48 \mathrm{~d}$ & $51.22 \pm 2.42 \mathrm{~cd}$ \\
TGML $10 \mathrm{mM}$ & $91.58 \pm 1.03 \mathrm{e}$ & $85.22 \pm 0.45 \mathrm{e}$ \\
SB $1.25 \mathrm{mM}$ & $3.51 \pm 1.24 \mathrm{a}$ & $8.97 \pm 1.21 \mathrm{a}$ \\
SB $2.5 \mathrm{mM}$ & $17.11 \pm 2.79 \mathrm{ab}$ & $14.21 \pm 1.89 \mathrm{a}$ \\
SB $5 \mathrm{mM}$ & $43.13 \pm 0.93 \mathrm{c}$ & $24.57 \pm 1.21 \mathrm{ab}$ \\
SB $10 \mathrm{mM}$ & $56.43 \pm 1.24 \mathrm{~d}$ & $42.41 \pm 1.44 \mathrm{c}$ \\
PS $1.25 \mathrm{mM}$ & $6.58 \pm 0.93 \mathrm{a}$ & $10.47 \pm 1.36 \mathrm{a}$ \\
PS $2.5 \mathrm{mM}$ & $22.08 \pm 2.38 \mathrm{ab}$ & $16.24 \pm 1.66 \mathrm{ab}$ \\
PS $5 \mathrm{mM}$ & $46.35 \pm 1.14 \mathrm{~cd}$ & $30.13 \pm 2.12 \mathrm{bc}$ \\
PS $10 \mathrm{mM}$ & $62.57 \pm 0.21 \mathrm{~d}$ & $45.09 \pm 2.27 \mathrm{~cd}$ \\
\hline
\end{tabular}

The mean values that are not followed by the same letter are significantly different $(p \leq 0.05)$.

incubated with allyl isothiocyanate at $0.1,1$, and $10 \mu \mathrm{l} / 1$ [1]. It was speculated that the inhibition of mycotoxin secretion might be due to the fact that the changes in the physiological environment of fungi affected the signal transduction of toxin biosynthesis [39]. Of course, the specific inhibitory mechanism still needed further exploration.

In conclusion, TGML was synthesized by esterification of triglycerol and lauric acid catalyzed by Lipozyme 435 . The highest TGML content (49.76\%) was obtained under the following conditions: triglycerol/lauric acid molar ratio $(1.08)$, reaction temperature $\left(84.93^{\circ} \mathrm{C}\right)$, reaction time $(6 \mathrm{~h})$ and enzyme dosage $(1.32 \%)$. It was purified to $98.3 \%$ by molecular distillation in combination with the washes of ethyl acetate and water. Its structure was proved to be linear triglycerol combined with lauroyl at the end by ESI-MS, IR and NMR. Besides, compared to SB and PS, TGML had a better inhibitory effects on the growth and toxin secretion of A. flavus and A. parasiticus. Therefore, TGML may be used as a substitute for traditional antifungal agents in the food industry.

\section{Acknowledgments}

This work was funded by the National Key R\&D Plan of China (No. 2018YFC1602102), the Fundamental Research Funds for the Central Universities and Dongguan Social Science and Technology Development (Key) Project (No. 2019507163160).

\section{Conflicts of Interest}

The authors have no financial conflicts of interest to declare.

\section{References}

1. Nazareth TM, Bordin K, Manyes L, Meca G, Manes J, Luciano FB. 2016. Gaseous allyl isothiocyanate to inhibit the production of aflatoxins, beauvericin and enniatins by Aspergillus parasiticus and Fusarium poae in wheat flour. Food Control. 62: 317-321.

2. Siahmoshteh F, Hamidi-Esfahani Z, Spadaro D, Shams-Ghahfarokhi M, Razzaghi-Abyaneh M. 2018. Unraveling the mode of antifungal action of Bacillus subtilis and Bacillus amyloliquefaciens as potential biocontrol agents against aflatoxigenic Aspergillus parasiticus. Food Control. 89: 300-307.

3. Cabral LD, Pinto VF, Patriarca A. 2013. Application of plant derived compounds to control fungal spoilage and mycotoxin production in foods. Int. J. Food Microbiol. 166: 1-14.

4. Moss MO. 2008. Fungi, quality and safety issues in fresh fruits and vegetables. J. Appl Microbiol. 104: 1239-1243.

5. Hohler D. 1998. Ochratoxin A in food and feed: occurrence, legislation and mode of action. Z. Ernahrungswiss 37: 2-12.

6. Suarez-Quiroz ML, Campos AA, Alfaro GV, Gonzalez-Rios O, Villeneuve P, Figueroa-Espinoza MC. 2013. Anti-Aspergillus activity of green coffee 5-O-caffeoyl quinic acid and its alkyl esters. Microb. Pathogenesis. 61-62: 51-56.

7. Williams JH, Phillips TD, Jolly PE, Stiles JK, Jolly CM, Aggarwal D. 2004. Human aflatoxicosis in developing countries: a review of toxicology, exposure, potential health consequences, and interventions. Am. J. Clin. Nutr. 80: 1106-1122.

8. Oliveira PM, Zannini E, Arendt EK. 2014. Cereal fungal infection, mycotoxins, and lactic acid bacteria mediated bioprotection: From crop farming to cereal products. Food Microbiol. 37: 78-95.

9. Mishra PK, Singh P, Prakash B, Kedia A, Dubey NK, Chanotiya CS. 2013. Assessing essential oil components as plant-based preservatives against fungi that deteriorate herbal raw materials. Int. Biodeterior. Biodegradation 80: 16-21.

10. Ma MM, Wen XF, Xie YT, Guo Z, Zhao RB, Yu P, et al. 2018. Antifungal activity and mechanism of monocaprin against food spoilage fungi. Food Control. 84: 561-568.

11. Luo CY, Zeng ZL, Gong DM, Zhao CY, Liang QF, Zeng C. 2014. Evaluation of monolaurin from camphor tree seeds for controlling food spoilage fungi. Food Control. 46: 488-494.

12. Altieri C, Bevilacqua A, Cardillo D, Sinigaglia M. 2009. Antifungal activity of fatty acids and their monoglycerides against Fusarium spp. in a laboratory medium. Int. J. Food Sci. Tech. 44: 242-245.

13. Mueller EA, Schlievert PM. 2015. Non-Aqueous glycerol monolaurate gel exhibits antibacterial and anti-biofilm activity against gram-positive and gram-negative pathogens. PLoS One 10(3): e0120280.

14. Zhang H, Taxipalati M, Yu LY, Que F, Feng FQ. 2013. Structure activity relationship of a U-type antimicrobial microemulsion system. PLoS One. 8: e76245. 
15. Obonyo M, Zhang L, Thamphiwatana S, Pornpattananangkul D, Fu V, Zhang LF. 2012. Antibacterial activities of liposomal linolenic acids against antibiotic-resistant helicobacter pylori. Mol. Pharmaceut. 9: 2677-2685.

16. Umerska A, Cassisa V, Matougui N, Joly-Guillou ML, Eveillard M, Saulnier P. 2016. Antibacterial action of lipid nanocapsules containing fatty acids or monoglycerides as co-surfactants. Eur. J. Pharm. Biopharm. 108: 100-110.

17. Kato T, Nakamura T, Yamashita M, Kawaguchi M, Kato T, Itoh T. 2003. Surfactant properties of purified polyglycerol monolaurates. J. Surfactants Deterg. 6: 331-337.

18. Tan CP, Nakajima M. 2005. Effect of polyglycerol esters of fatty acids on physicochemical properties and stability of beta-carotene nanodispersions prepared by emulsification/evaporation method. J. Sci. Food Agr. 85: 121-126.

19. Shimazaki A, Sakamoto JJ, Furuta M, Tsuchido T. 2016. Antifungal activity of diglycerin ester of fatty acids against yeasts and its comparison with those of sucrose monopalmitate and sodium benzoate. Biocontrol. Sci. 21: 123-130.

20. Ikegawa C, Ogita A, Doi T, Kumazawa F, Fujita KI, Tanaka T. 2017. Involvement of irreversible vacuolar membrane fragmentation in the lethality of food emulsifier diglycerol monolaurate against budding teast. J. Agric. Food Chem. 65: 5650-5656.

21. Zhang S, Xiong J, Lou W, Ning Z, Zhang D, Yang J. 2019. Antimicrobial activity and action mechanism of triglycerol monolaurate on common foodborne pathogens. Food Control. 98: 113-119.

22. Kumar TN, Sastry YSR, Lakshminarayana G. 1989. Preparation and surfactant properties of diglycerol esters of fatty acids. J. Am. Oil Chem. Soc. 66: 153-157.

23. Wang WY, Liu CY, Zhang GJ, Yang F, Wang XY, Chen FL, et al. 2019. Green synthesis of decaglycerol laurates by lipase-catalyzed transesterification of methyl laurate with decaglycerol. J. Chem. Article ID 6342475

24. Denecke P, Börner G, Allmen V. 1981. Method of preparing polyglycerol polyricinoleic fatty acid esters. UK Patent. 2: 232.

25. Ortega-Requena S, Bodalo-Santoyo A, Bastida-Rodriguez J, Maximo-Martin MF, Montiel-Morte MC, Gomez-Gomez M. 2014. Optimized enzymatic synthesis of the food additive polyglycerol polyricinoleate (PGPR) using Novozym (R) 435 in a solvent free system. Biochem. Eng. J. 84: 91-97.

26. Wan FL, Teng YL, Wang Y, Li AJ, Zhang N. 2015. Optimization of oligoglycerol fatty acid esters preparation catalyzed by Lipozyme 435. Grasas Aceites. 66(3): e088.

27. Conley AJ, Kabara JJ. 1973. Antimicrobial action of esters of polyhydric alcohols. Antimicrob. Agents. Chemother. 4: 501-506.

28. Holstborg J, Pedersen BV, Krog N, Olesen SK. 1999. Physical properties of diglycerol esters in relation to rheology and stability of protein-stabilised emulsions. Colloid Surface B. 12: 383-390.

29. Peng B, Xiong CY, Huang Y, Hu JN, Zhu XM, Deng ZY. 2018. Enzymatic synthesis of polyglycerol fatty acid esters and their application as emulsion stabilizers. J. Agric. Food Chem. 66: 8104-8113.

30. REN Chun-fang, ZHOU Li-guo, Dian-qing Y. 2007. The improvement of method determining hydroxyl value in polyglycerol. J. Qilu. Univ. Technol. (Natural Science Edition) 21: 54-55.

31. Martins M, Klusczcovski AM, Scussel VM. 2014. In vitro activity of the Brazil nut (Bertholletia excelsa HBK) oil in aflatoxigenic strains of Aspergillus parasiticus. Eur. Food Res. Technol. 239: 687-693.

32. Shao S, Cai J, Du X, Wang CG, Lin JG, Dai J. 2016. Biotransformation and detoxification of aflatoxin B-1 by extracellular extract of Cladosporium uredinicola. Food Sci. Biotechnol. 25: 1789-1794.

33. Eom TK, Kong CS, Byun HG, Jung WK, Kim SK. 2010. Lipase catalytic synthesis of diacylglycerol from tuna oil and its anti-obesity effect in C57BL/6J mice. Process Biochem. 45: 738-743.

34. Orfanakis A, Hatzakis E, Kanaki K, Pergantis SA, Rizos A, Dais P. 2013. Characterization of polyglycerol polyricinoleate formulations using NMR spectroscopy, mass spectrometry and dynamic light scattering. J. Am. Oil Chem. Soc. 90: 39-51.

35. Wan FL, Teng YL, Zou L, Yang X, Chen Q, Li AJ, et al. 2016. Effect of addition of purified diglycerol linoleic acid esters on the crystallization behavior of diacylglycerol oils. J. Am. Oil Chem. Soc. 93: 1605-1614.

36. Ma X, Yan RA, Yu SQ, Lu YY, Li Z, Lu HH. 2012. Enzymatic acylation of isoorientin and isovitexin from bamboo-leaf extracts with fatty acids and antiradical activity of the acylated derivatives. J. Agric. Food Chem. 60: 10844-10849.

37. Saladino F, Bordin K, Manyes L, Luciano FB, Manes J, Fernandez-Franzon M, et al. 2016. Reduction of the aflatoxins B-1, B-2, G(1) and G(2) in Italian piadina by isothiocyanates. Lwt-Food Sci. Technol. 70: 302-308.

38. Saladino F, Manyes L, Luciano FB, Manes J, Fernandez-Franzon M, Meca G. 2016. Bioactive compounds from mustard flours for the control of patulin production in wheat tortillas. Lwt-Food Sci. Technol. 66: 101-107.

39. Holmes RA, Boston RS, Payne GA. 2008. Diverse inhibitors of aflatoxin biosynthesis. Appl. Microbiol. Biotechnol. 78: 559-572. 